\title{
Emodin Alleviates Liver Fibrosis of Mice by Reducing Infiltration of $\mathrm{Gr}^{\mathrm{hi}}$ Monocytes
}

\author{
Xiang-An Zhao ${ }^{10},{ }^{1}$ Guangmei Chen, ${ }^{2}$ Yong Liu, ${ }^{3}$ Hongyan Wu, ${ }^{4}$ Jin Chen, ${ }^{4}$ Yali Xiong, ${ }^{5}$ \\ Chen Tian, ${ }^{5}$ Bei Jia, ${ }^{5}$ Guiyang Wang, ${ }^{5}$ Juan Xia, ${ }^{5}$ Yuxin Chen, ${ }^{3}$ Jian Wang, ${ }^{6}$ Xiaomin Yan, \\ Zhaoping Zhang, ${ }^{5}$ Rui Huang, ${ }^{5}$ and Chao $W u$ iD ${ }^{1,5}$ \\ ${ }^{1}$ Department of Infectious Diseases, Nanjing Drum Tower Hospital, Clinical College of Traditional Chinese and Western Medicine, \\ Nanjing University of Chinese Medicine, Nanjing, Jiangsu, China \\ ${ }^{2}$ Department of Infectious Diseases, Affiliated Hospital of Nanjing University of Traditional Chinese Medicine, Nanjing, Jiangsu, China \\ ${ }^{3}$ Department of Laboratory Medicine, Nanjing Drum Tower Hospital, The Affiliated Hospital of Nanjing University Medical School, \\ Nanjing, Jiangsu, China \\ ${ }^{4}$ Department of Pathology, Nanjing Drum Tower Hospital, The Affiliated Hospital of Nanjing University Medical School, \\ Nanjing, Jiangsu, China \\ ${ }^{5}$ Department of Infectious Diseases, Nanjing Drum Tower Hospital, The Affiliated Hospital of Nanjing University Medical School, \\ Nanjing, Jiangsu, China \\ ${ }^{6}$ Department of Infectious Diseases, Nanjing Drum Tower Hospital, Clinical College of Nanjing Medical University, \\ Nanjing, Jiangsu, China
}

Correspondence should be addressed to Chao Wu; dr.wu@nju.edu.cn

Received 4 August 2017; Revised 14 December 2017; Accepted 1 January 2018; Published 21 March 2018

Academic Editor: Eman Al-Sayed

Copyright (C) 2018 Xiang-An Zhao et al. This is an open access article distributed under the Creative Commons Attribution License, which permits unrestricted use, distribution, and reproduction in any medium, provided the original work is properly cited.

Emodin, as a major active component of Rheum palmatum L. and Polygonum cuspidatum, has been reported to have antifibrotic effect. However, the mechanism of emodin on antifibrotic effect for liver fibrosis was still obscure. In the present study, we aimed to investigate whether emodin can alleviate carbon tetrachloride- $\left(\mathrm{CCl}_{4^{-}}\right)$induced liver fibrosis through reducing infiltration of $\mathrm{Gr}^{\mathrm{hi}}$ monocytes. Liver fibrosis was induced by intraperitoneal $\mathrm{CCl}_{4}$ injection in mice. Mice in the emodin group received emodin treatment by gavage. Pretreatment with emodin significantly protected mice from liver inflammation and fibrosis revealed by the decreased elevation of serum alanine aminotransferase (ALT) and aspartate aminotransferase (AST), as well as reduced hepatic necrosis and fibrosis by analysis of hematoxylin-eosin (HE) staining, Masson staining, $\alpha$-smooth muscle actin $(\alpha$-SMA), and collagen-I immunohistochemistry staining. Further, compared to $\mathrm{CCl}_{4}$ group, mice in the emodin group showed significantly less intrahepatic infiltration of $\mathrm{Gr}^{\mathrm{hi}}$ monocytes. Moreover, emodin significantly inhibited hepatic expression of interleukin-1 $\beta$ (IL-1 $\beta$ ), tumor necrosis factor- $\alpha$ (TNF- $\alpha$ ), transforming growth factor- $\beta 1$ (TGF- $\beta 1$ ), granulin (GRN), monocyte chemoattractant protein 1 (MCP-1), and chemokine ligand 7 (CCL7), which was in line with the decreased numbers of intrahepatic Gr1 ${ }^{\text {hi }}$ monocytes. In conclusion, emodin can alleviate the degree of liver fibrosis by reducing infiltration of $\mathrm{Gr} 1^{\text {hi }}$ monocytes. These results suggest that emodin is a promising candidate to prevent and treat liver fibrosis.

\section{Introduction}

Liver fibrosis, characterized by excessive deposition of extracellular matrix (ECM), is a pathological change after chronic liver injury including viral infection, toxic damage, metabolic disorders, and alcohol abuse [1]. However, there is still a lack of effective drugs for the treatment of liver fibrosis [2].
Understanding the mechanisms of liver fibrosis is important for prevention and control of liver disease progression.

Different cell types in the liver make a network and regulate liver fibrogenesis [3]. During the past decades, it became apparent that hepatic macrophages play central functions in initiating, perpetuating, and restricting liver fibrosis [1, 4-6]. Hepatic macrophages participate in 
liver fibrosis through various ways. Importantly, hepatic macrophages mediate the transdifferentiation of hepatic stellate cells (HSCs) into collagen-producing myofibroblasts by secreting several profibrotic factors, such as transforming growth factor- $\beta 1$ (TGF- $\beta 1$ ), platelet-derived growth factor (PDGF), and granulin (GRN) in liver fibrosis [1, 5, 7-9]. Besides, hepatic macrophages can release many proinflammatory cytokines such as tumor necrosis factor- $\alpha$ (TNF$\alpha$ ), interleukin-1 $\beta$ (IL-1 $\beta$ ), and IL-6 which may induce liver cellular apoptosis and aggravate liver inflammation $[8,10,11]$. Thus, hepatic macrophages are an attractive target for novel therapeutic approaches to liver fibrosis [12].

Hepatic macrophages comprise resident Kupffer cells (KFs) and infiltrating monocyte-derived cells [13]. During the development of liver fibrosis, macrophage pool of the liver can be rapidly expanded by infiltrating phagocytes that mainly originate from circulating monocytes [14-16]. In mice, two major populations of circulating monocytes exist: $\mathrm{Gr}^{\text {hi }}\left(\right.$ Ly-6C $\left.\mathrm{C}^{\text {hi }}\right)$ monocytes and Gr1 ${ }^{\text {lo }}\left(\right.$ Ly-6C $\left.{ }^{\text {lo }}\right)$ monocytes. In acute and chronic liver injury of mice, $\mathrm{Gr} 1^{\text {hi }}$ monocytes are massively recruited into the liver $[8,11]$. As vigorous secretors of proinflammatory and profibrotic factors including TNF$\alpha$ and TGF- $\beta 1$, they drive inflammation and activate HSC thereby triggering a cascade of events leading to liver fibrosis $[10,17]$.

The accumulation of monocytes is critically due to chemokines monocyte chemoattractant protein 1 (MCP-1) and chemokine ligand 7 (CCL7) $[8,18-20]$. When the liver is damaged, liver cells, HSCs, macrophages, and endothelial cells can secrete MCP-1 and CCL7 which combine with the related receptor-chemokine receptor on peripheral blood monocytes surface and recruit $\mathrm{Gr} 1^{\mathrm{hi}}$ monocytes into liver $[8,21,22]$.

In the past two thousand years, Rheum palmatum L. and Polygonum cuspidatum has been used as Traditional Chinese Medicine to treat liver diseases, which has achieved significant curative effects [23]. As the main active ingredient of Rheum palmatum L. and Polygonum cuspidatum, emodin has been demonstrated to have anti-inflammatory, antitumor, and antifibrotic effects [24,25]. Further study suggested that emodin has certain antifibrotic effect on liver fibrosis [26, 27]. However, the mechanism of emodin on the antifibrotic effect for liver fibrosis is still obscure. Therefore, in the present study we investigated whether emodin could alleviate the degree of carbon tetrachloride- $\left(\mathrm{CCl}_{4}{ }^{-}\right)$induced liver fibrosis by inhibiting infiltration of $\mathrm{Grl}^{\text {hi }}$ monocytes.

\section{Materials and Methods}

2.1. Mice. Male C57BL/6 mice, 7-8 weeks old, weighing $25 \pm 2 \mathrm{~g}$, purchased from Beijing Vital River Experimental Animals Technology (Beijing, China), were used in this study. Mice were housed under laminar airflow hoods in a specific pathogen-free room with a $12 \mathrm{~h}$ light and $12 \mathrm{~h}$ dark schedule and fed autoclaved chow and water at a controlled temperature of $22 \pm 2^{\circ} \mathrm{C}, 50-60 \%$ relative humidity.
2.2. Experimental Protocol. Mice were randomly assigned to the control group, $\mathrm{CCl}_{4}$ group, and emodin group. For induction of liver fibrosis, mice in the emodin group and $\mathrm{CCl}_{4}$ group were injected intraperitoneally with $0.6 \mathrm{ml} / \mathrm{kg}$ dose of $\mathrm{CCl}_{4}\left(\mathrm{CCl}_{4}\right.$ : olive oil $=1: 4,3 \mu \mathrm{l} / \mathrm{g} \mathrm{CCl}_{4}$ oil $)$ twice weekly for 4 weeks. Mice in the control group were injected with equal volume of olive oil as control. Emodin was dissolved in $0.25 \%$ sodium carboxymethyl cellulose (CMC-Na) aqueous solution to prepare the appropriate concentration. Emodin was given by gavage at a dose of $20 \mathrm{mg} / \mathrm{kg} / \mathrm{d}$ in the emodin group. This dose of emodin is the optimal dose proved by the previous study [27]. For the control group and $\mathrm{CCl}_{4}$ group, mice were administrated orally with the same volume of CMC-Na aqueous solution.

After $48 \mathrm{~h}$ of the last $\mathrm{CCl}_{4}$ administration, mice were sacrificed after anesthesia. Blood samples were collected for ALT and AST detection. The liver tissues of mice were collected.

2.3. Liver Function Detection. Blood samples were centrifuged at $4000 \mathrm{rpm} / \mathrm{min}$ for $15 \mathrm{~min}$ to get serum. ALT and AST levels were determined with clinical testing technique through biochemical instrument (Beckman Coulter, Inc., Brea, CA, USA).

2.4. Flow Cytometry. Isolation of liver-infiltrating lymphocytes was performed by an automated, mechanical disaggregation system (Medimachine, Becton Dickinson, USA). The liver samples of mice were washed with PBS twice and then cut into small pieces of $3-4 \mathrm{~mm}^{3}$. Five pieces were immediately put in a disposable disaggregator Medicon with $50 \mu \mathrm{m}$ separator mesh (Becton Dickinson, USA) and then $1 \mathrm{ml}$ PBS was added and processed in the Medimachine System for $1 \mathrm{~min}$. Disaggregated cells were removed and pressed through $70 \mu \mathrm{m}$ cell strainers to obtain single cell suspensions. Cells were used immediately for flow cytometric analysis. Related antibodies were listed as follows: CD45 (557235, BD Pharmingen, USA), CD11b (557397, BD Pharmingen, USA), Gr1/Ly6C (560595, BD Pharmingen, USA), Ly6G (551460, BD Pharmingen, USA), and F4/80 (25-4801, eBioscience, USA). Flow cytometric analysis was performed on a FACS Aria II (BD Bioscience, USA).

2.5. Histopathology. Mouse liver tissues were fixed in paraformaldehyde for $48 \mathrm{~h}$, then dehydrated, and finally embedded with paraffin. Paraffin-embedded liver samples were sectioned to $3 \mu \mathrm{m}$ thin slices, which were performed with hematoxylin-eosin (HE) staining and Masson staining according to standard protocols.

2.6. Immunohistochemistry. $3 \mu \mathrm{m}$ paraffin sections of mouse liver samples were dewaxed and hyalinized. Endogenous peroxidase was blocked with $3 \% \mathrm{H}_{2} \mathrm{O}_{2}$. Antigen repair was achieved by boiling in ethylenediaminetetraacetic acid(EDTA-) alkaline solution. Then, sample sections were incubated with various primary antibody: $\alpha$-smooth muscle actin ( $\alpha$-SMA, ab5694, Abcam, USA), collagen-I (ab34710, Abcam, USA), F4/80 (ab111101, Abcam, USA), CD45 (ab10558, Abcam, USA), CD11b (ab13357, Abcam, USA), TGF- $\beta 1$ 
TABLE 1: Sequences of primers used for real-time PCR [28].

\begin{tabular}{lcc}
\hline Gene & Direction & Primer sequence $\left(5^{\prime}-3^{\prime}\right)$ \\
\hline TGF- $\beta 1$ & Forward & GTGGAAATCAACGGGATCAG \\
& Reverse & ACTTCCAACCCAGGTCCTTC \\
GRN & Forward & GCTACAGACTTAAGGAACTC \\
& Reverse & GAAATGGCAGTTTGATACGG \\
MCP-1 & Forward & ATTGGGATCATCTTGCTGGT \\
& Reverse & CCTGCTGTTCACAGTTGCC \\
CCL7 & Forward & CGTCCCGTAGACAAAATGGTGAA \\
& Reverse & GCCGTGAGTGGAGTCATACTGGAACA \\
IL-1 $\beta$ & Forward & GGTCAAAGGTTTGGAAGCAG \\
& Reverse & TGTGAAATGCCACCTTTTGA \\
IL-6 & Forward & CATTTCCACGATTTCCCAGA \\
TNF- $\alpha$ & Reverse & TCCCTCTGTGATCTGGGAAG \\
$\beta$-Actin & Forward & AGGGTCTGGGCCATAGAACT \\
& Reverse & CCACCACGCTCTTCTGTCTAC \\
\hline
\end{tabular}

(ab92486, Abcam, USA), GRN (ab191211, Abcam, USA), MCP-1 (ab25124, Abcam, USA), or CCL7 (orb315556, Biorbyt, $\mathrm{UK})$ overnight at $4^{\circ} \mathrm{C}$. Then the sections were stained with secondary antibody (Life Technologies, USA) after multiple flushing with PBS buffer. At last, generally diaminobenzidine (DAB) stained, haematoxylin slightly stained, and neutral balata fixed.

2.7. Histological and Immunohistochemical Evaluation. Sections with HE staining were examined under light microscopy by an experienced pathologist in a blinded fashion for liver steatosis, necrosis, and leukocyte infiltration.

For Masson staining and immunohistochemical staining of $\alpha$-SMA and collagen-I, five $\times 100$ magnification images of Masson staining and immunohistochemical staining were captured in each section. Positive staining area of Masson staining (blue), $\alpha$-SMA, or collagen-I immunohistochemical staining (brown) per high-power field (HPF) of each image was converted into pixels by Image-ProPlus software (Media Cybernetics, USA). The percentage of positive area was expressed as a fraction of the total number of pixels, averaged across the 5 different regions per section.

Absolute counts of CD $45^{+}$cells (leucocytes), F4/80 ${ }^{+}$cells (macrophages), and $\mathrm{CD} 1 \mathrm{~b}^{+}$cells (monocytes) per HPF of stained liver sections were manually assessed in 5 different fields per section. All quantification was carried out blinded and without prior knowledge of sections or groups.

2.8. Real-Time Gene Expression Analysis. Total RNA was extracted from $50 \mathrm{mg}$ frozen liver tissues using TRIzol according to the manufacturer's instructions (Life Technologies, USA) and then reverse-transcribed to get cDNA by using the PrimeScript RT Master Mix kit (Takara, China) according to the relevant experimental manual. Real-time PCR was performed on Step One Plus Real-Time PCR Systems (Life Technologies, USA) using SYBR Premix Ex Taq kit (Takara, China). All primers and PCR product sizes of this study are listed in Table $1 . \beta$-Actin was used as an internal control [28].

2.9. Statistical Analysis. All data are expressed as the mean \pm standard error of the mean (SEM). Statistical analysis was performed using one-way analysis of variance (ANOVA) test by SPSS 22.0 software. $P<0.05$ was considered to be statistically significant.

\section{Results}

3.1. Emodin Attenuated $\mathrm{CCl}_{4}$-Caused Liver Inflammation. The protective effect of emodin on $\mathrm{CCl}_{4}$-induced liver injury was identified by liver function and HE staining. As shown in Figure 1(a), the serum ALT and AST increased significantly in mice of $\mathrm{CCl}_{4}$ group as compared with the control group. Emodin can significantly reduce the increased liver enzymes. The liver tissues of mice in the $\mathrm{CCl}_{4}$ group showed liver steatosis, necrosis, and leukocyte infiltration which were significantly relieved in the emodin group (Figure 1(b)). These results demonstrated that emodin can significantly reduce $\mathrm{CCl}_{4}$-induced liver inflammation in vivo.

3.2. Emodin Alleviated Liver Fibrosis Induced by $\mathrm{CCl}_{4}$. Masson staining was used to observe the collagen fibers of liver tissues. As shown in Figure 2, fiber staining only appeared on the central venous wall, part of portal area, and interlobular septa in hepatic lobules of control mice. However, compared to the control group, the collagen fibers in $\mathrm{CCl}_{4}$ group were significantly higher. The initial formation of interlobular septa and significantly increased portal fiber deposition were observed. The liver tissues of mice in the emodin group also had fiber deposition, mainly distributed at the portal area, fibrous septum, and central vein. But the density of 

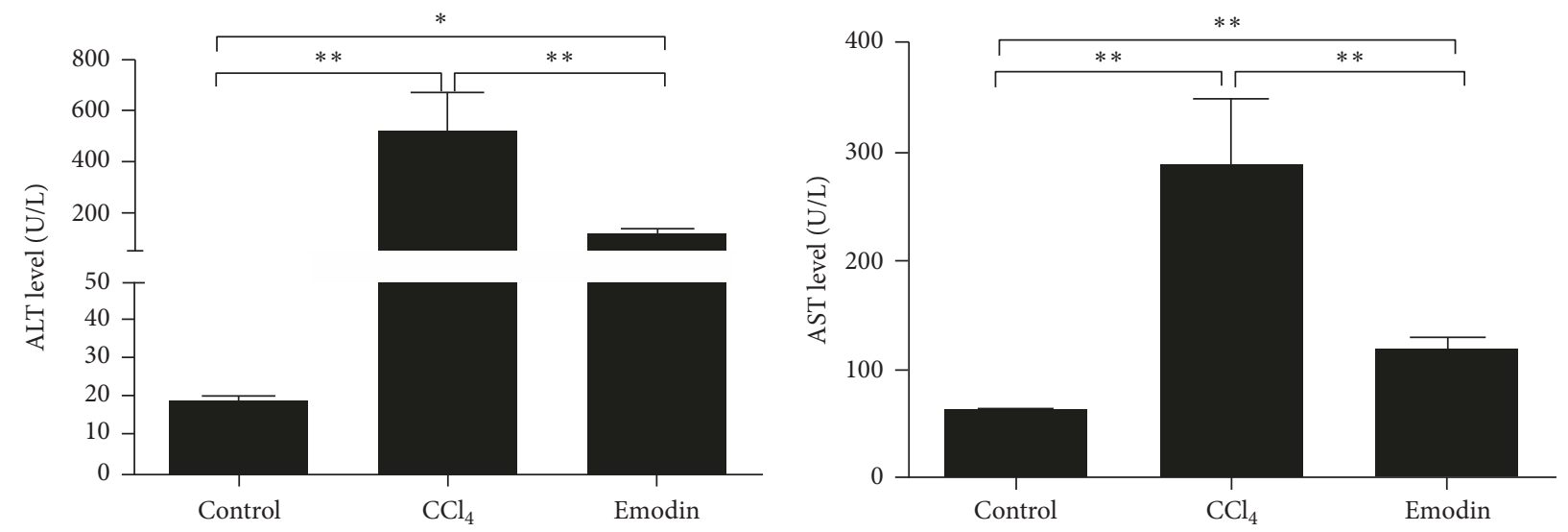

(a)
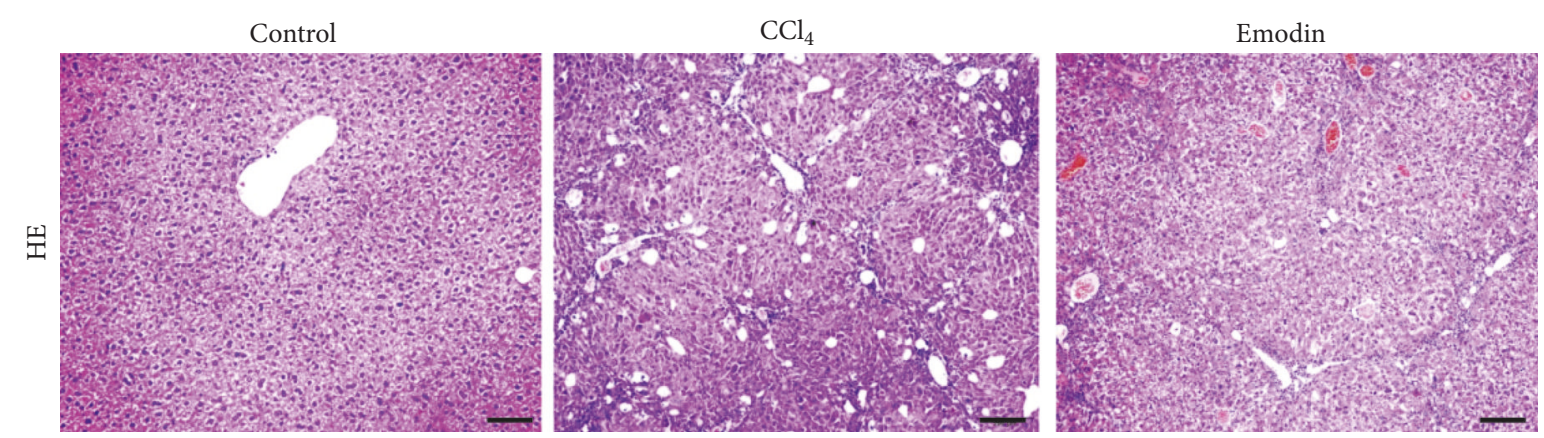

(b)

FIGURE 1: Emodin attenuated $\mathrm{CCl}_{4}$-caused liver inflammation. (a) ALT and AST levels of mice in each group. (b) Hematoxylin-eosin staining of the liver tissues. All data are expressed as the mean \pm SEM. ${ }^{*} P<0.05 ;{ }^{* *} P<0.01$. Original magnification: $\times 100$; bar $=50 \mu \mathrm{m}$.

collagen deposition was significantly less than that of $\mathrm{CCl}_{4}$ group. Moreover, the liver tissues of mice in the control group had only a small amount of $\alpha$-SMA and collagenI expression (Figure 2). However, the hepatic expression of $\alpha$-SMA and collagen-I significantly increased in the $\mathrm{CCl}_{4}$ group (Figure 2). Emodin treatment can significantly reduce the expression of $\alpha$-SMA and collagen-I induced by $\mathrm{CCl}_{4}$ (Figure 2). These results collectively demonstrated that emodin can significantly reduce $\mathrm{CCl}_{4}$-induced liver fibrosis in vivo.

3.3. Emodin Reduced Gri ${ }^{\text {hi }}$ Monocyte Infiltration. After $\mathrm{CCl}_{4}$ challenge for 4 weeks, infiltrations of leukocytes, monocytes, and macrophages increased significantly in the mouse liver tissues of $\mathrm{CCl}_{4}$ group. However, compared to $\mathrm{CCl}_{4}$ group, mouse liver tissues of emodin group had greatly reduced infiltrations of leukocytes, monocytes, and macrophages (Figures 3(a) and 3(b)). The proportion of $\mathrm{Gr}^{\mathrm{hi}}$ monocyte

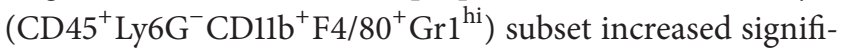
cantly in the liver tissues of $\mathrm{CCl}_{4}$ group as compared with the control group. However, the proportion of $\mathrm{Gr}^{\mathrm{hi}}$ monocyte subset was significantly lower in the emodin group compared to the $\mathrm{CCl}_{4}$ group (Figure 4(b)). These results suggested that emodin can significantly reduce infiltration of monocytederived macrophages, especially $\mathrm{Gr} 1^{\mathrm{hi}}$ monocyte in chronic liver injury.
3.4. Emodin Inhibited the Expression of $\mathrm{GrI}^{\text {hi }}$ Monocyte Associated Proinflammatory and Profibrogenic Cytokines. Gr $1^{\text {hi }}$ monocyte can promote liver fibrosis through releasing many proinflammatory and profibrotic cytokines $[1,5,7-9]$. As shown in Figures 5 and 6 , the intrahepatic mRNA expression of IL- $1 \beta$, IL- 6 , TNF- $\alpha$, TGF- $\beta 1$, and GRN significantly increased after $\mathrm{CCl}_{4}$ administration. Mice in the emodin group had significantly lower intrahepatic expression of IL$1 \beta$, TNF- $\alpha$, GRN, and TGF- $\beta 1$ as compared with $\mathrm{CCl}_{4}$ group. As shown in immunohistochemical staining, emodin group had significantly lower expression of TGF- $\beta 1$ and GRN (Figure 6).

3.5. Emodin Inhibited the Expression of MCP-1 and CCL7. MCP-1 and CCL7 are the prime monocyte chemotactic factor [8, 18-20]. Mice in $\mathrm{CCl}_{4}$ group have strongly increased hepatic expression of MCP-1 and CCL7, which may be responsible for increasing numbers of intrahepatic $\mathrm{Gr} \mathrm{h}^{\mathrm{hi}}$ monocytes (Figure 7). However, it is obvious that emodin effectively inhibited the hepatic expression of MCP-1 and CCL7 (Figure 7), which may explain why emodin could reduce the infiltration of monocytes.

\section{Discussion}

Hepatic macrophages, especially monocyte-derived macrophages, hold a central position in the pathogenesis of chronic 

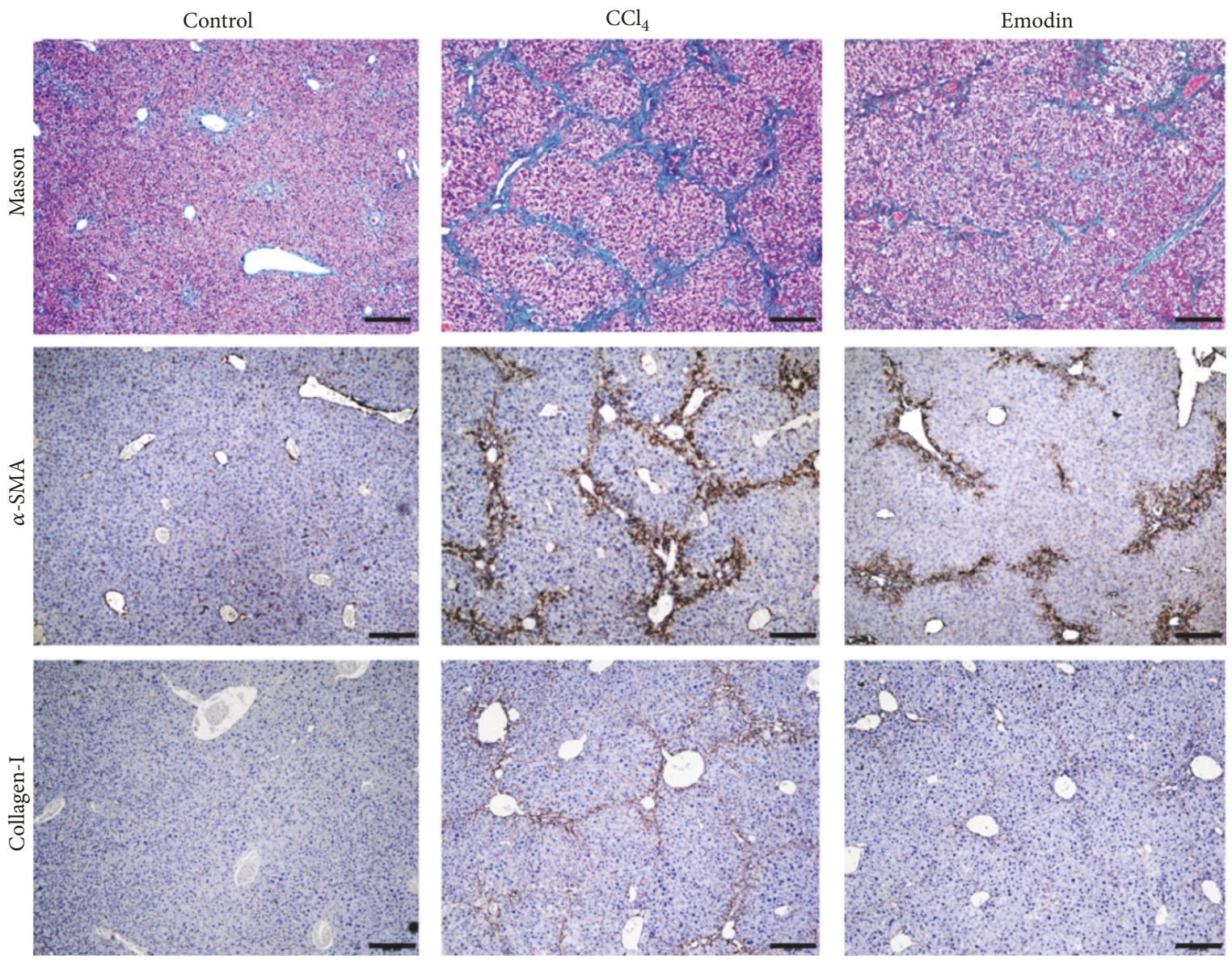

(a)
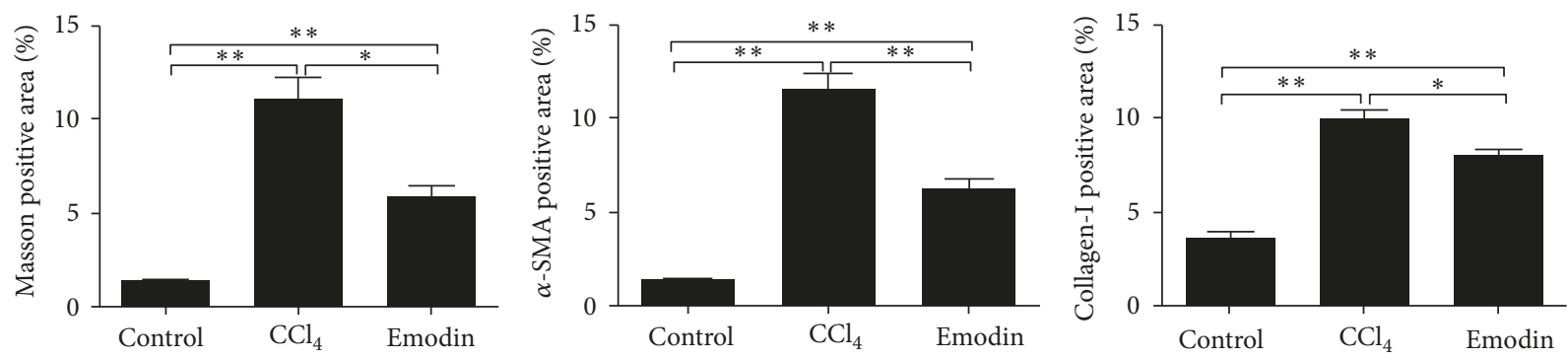

(b)

FIGURE 2: Emodin reduced $\mathrm{CCl}_{4}$-caused liver fibrosis in mice. (a) Masson staining, $\alpha$-SMA staining, and collagen-I staining of the liver tissues. (b) Statistical analyses of collagen deposition. All data are expressed as the mean \pm SEM. ${ }^{*} P<0.05 ;{ }^{* *} P<0.01$. Original magnification: $\times 100$; bar $=50 \mu \mathrm{m}$.

liver injury and have been proposed as potential targets for liver fibrosis [13, 22, 29]. In acute and chronic liver injury, $\mathrm{Grl}^{\mathrm{hi}}$ monocytes will firstly be recruited into inflammatory region and release proinflammatory and profibrogenic cytokines which will aggravate liver inflammation and fibrosis of liver [8,17]. In the present study, we confirmed that, after repeated $\mathrm{CCl}_{4}$ injections, $\mathrm{Gr} 1^{\text {hi }}$ monocytes were markedly increased in the liver tissues of mice.

Pharmacological inhibition of $\mathrm{Gr}^{\text {hi }}$ hepatic monocytes may be capable of limiting chronic liver injury and fibrosis in vivo [30]. Emodin, as a major active component of Rheum palmatum L. and Polygonum cuspidatum, has been reported to have anti-inflammatory, antitumor, and antifibrotic effect $[24,25]$. Emodin can ameliorate hepatic steatosis through endoplasmic reticulum-stress sterol regulatory element-binding protein 1c pathway in liquid fructosefeeding rats [31]. Liu et al. also reported that emodin could ameliorate liver steatosis and decrease hepatic triglyceride in mice fed with ethanol [32]. In addition, emodin could also significantly inhibit the growth of HSC-T6 cells in vitro [33]. Emodin can also protect the rat liver from $\mathrm{CCl}_{4}$ induced liver fibrosis by inhibiting HSCs activation in vivo 

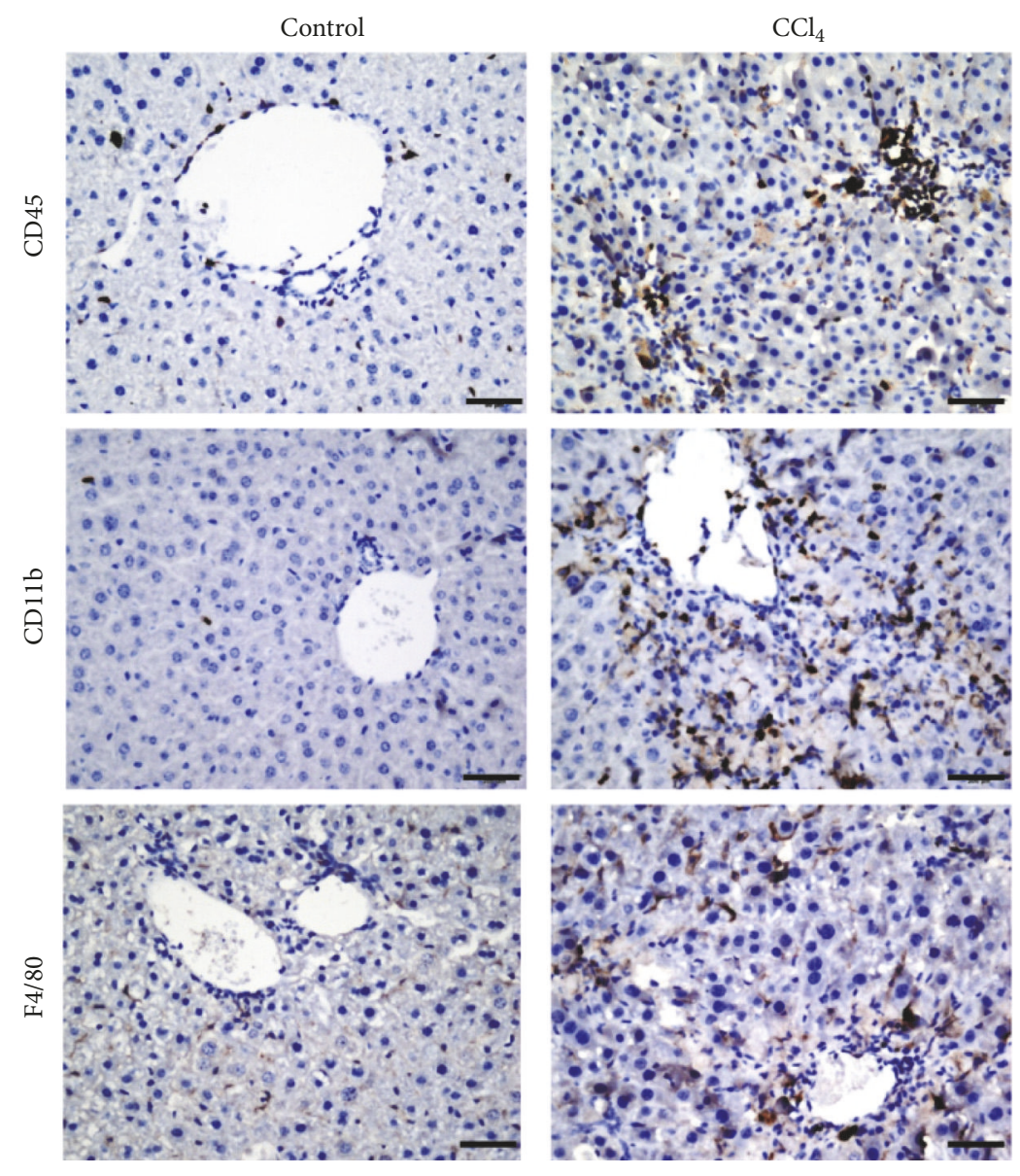

(a)
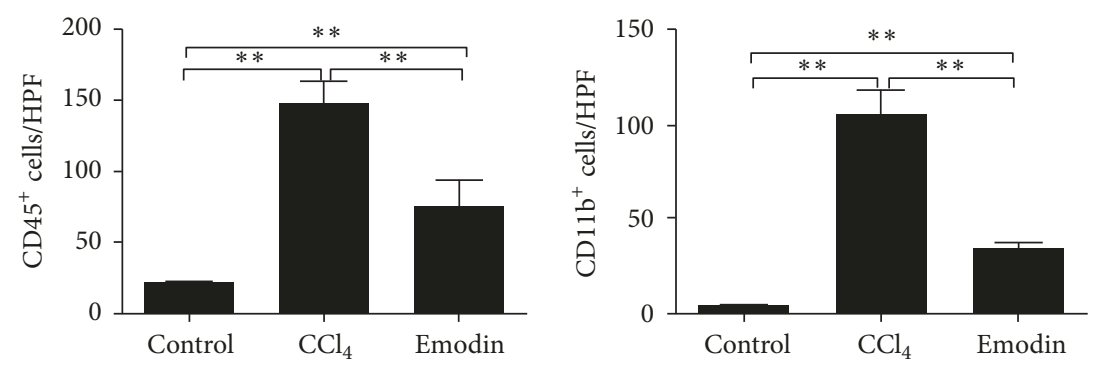

(b)
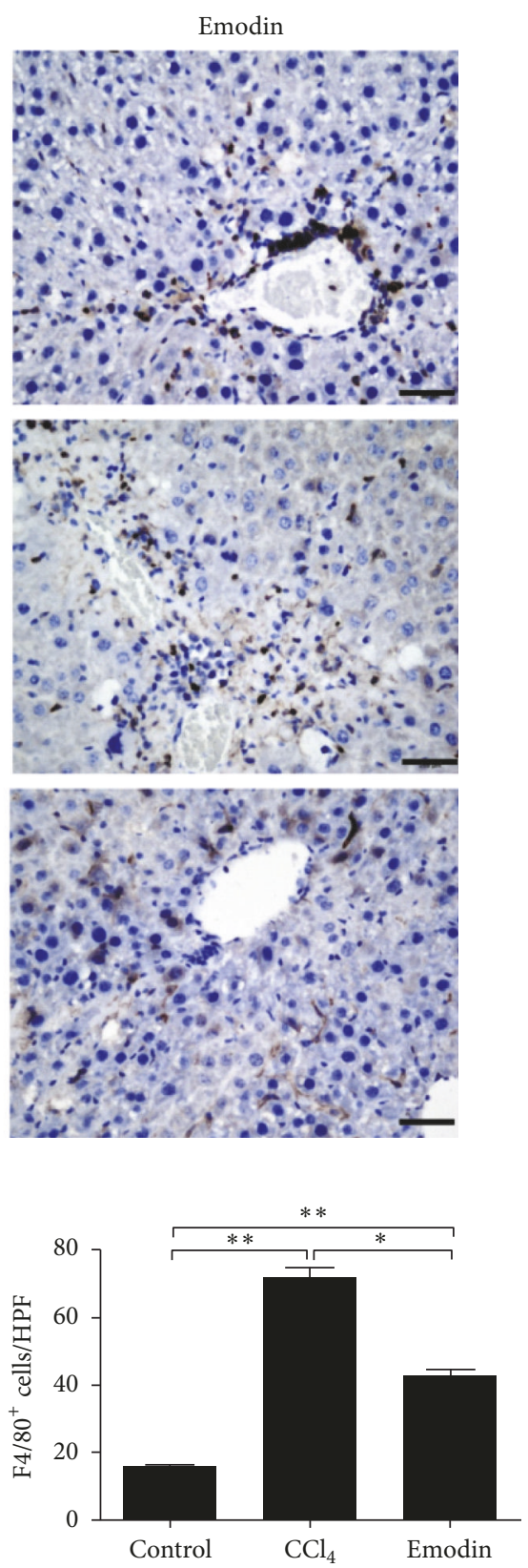

FIGURE 3: Emodin reduced infiltrations of leukocytes, monocytes, and macrophages in liver fibrosis. (a) Immunohistochemistry staining of leukocytes, monocytes, and macrophages in the liver tissues. (b) Statistical analyses of leukocytes, monocytes, and macrophages. All data are expressed as the mean \pm SEM. ${ }^{*} P<0.05 ;{ }^{* *} P<0.01$. Original magnification: $\times 400$; bar $=200 \mu \mathrm{m}$.

[27]. In the present study, we also confirmed that emodin could reduce liver inflammation and fibrosis in mouse model induced by $\mathrm{CCl}_{4}$. Recent studies show that emodin can inhibit homologous lymphotoxins-induced monocytes migration [34]. On this basis, we used immunohistochemistry staining and flow cytometry to observe the changes of monocytes, especially $\mathrm{Gr}^{\mathrm{hi}}$ monocytes. We found that emodin significantly reduced the infiltration of $\mathrm{Gr}^{\text {hi }}$ monocytes which is novel mechanism of the antifibrotic effect of emodin.
Gr1 ${ }^{\text {hi }}$ monocytes can release many proinflammatory and profibrogenic cytokines, including TNF- $\alpha$, IL-1 $\beta$, GRN, and TGF- $\beta 1$. TNF- $\alpha$ and IL- $1 \beta$ are mainly from monocytes and macrophages in the acute and chronic liver injury [35] and may trigger the production of many other proinflammatory cytokines and induce hepatocyte death through the recruitment of neutrophils $[36,37]$. TGF- $\beta 1$ is considered to be the most potent profibrogenic cytokine $[38,39]$. TGF- $\beta 1$ can promote liver fibrosis through multiple pathways, for example, activating HSCs, stimulating collagen gene transcription, and 

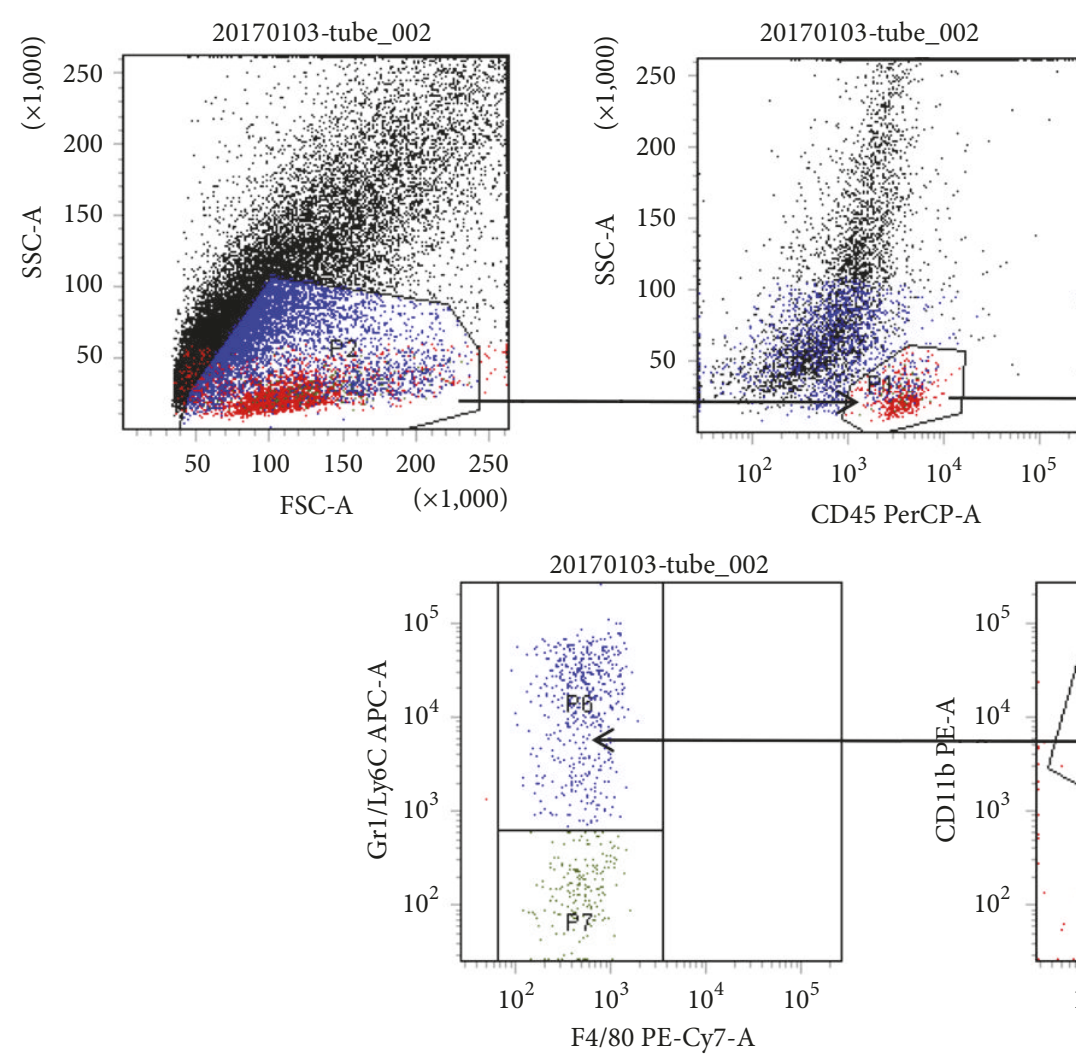

CD45 PerCP-A

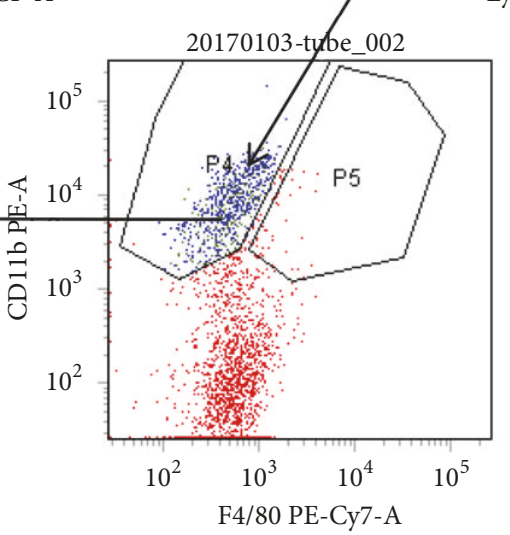

(a)
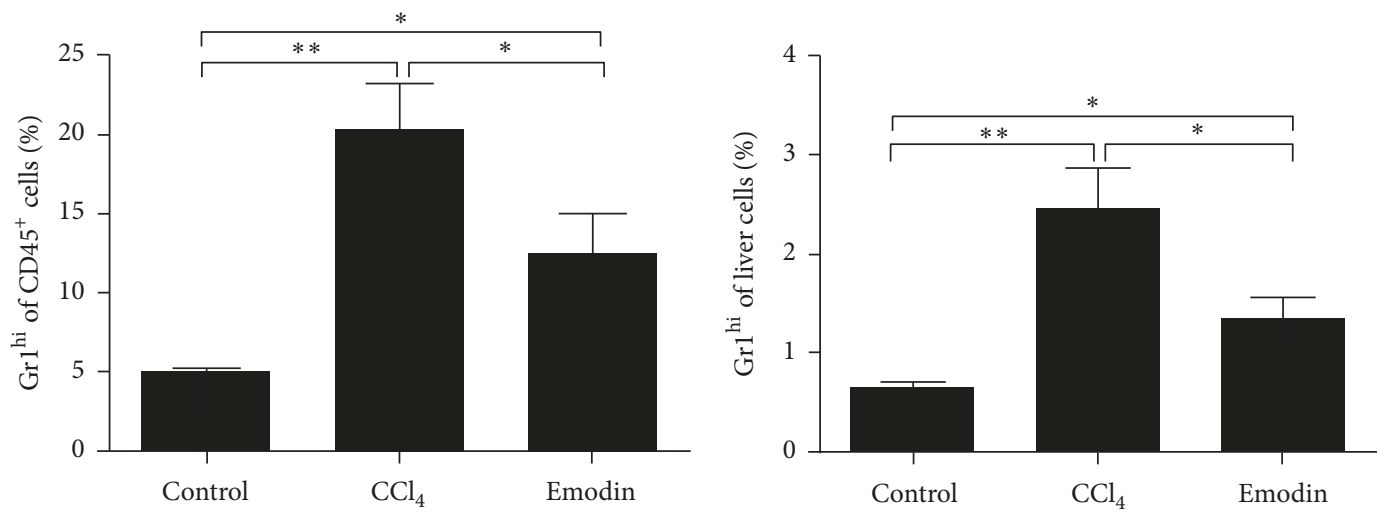

(b)

FIGURE 4: Emodin reduced infiltrations of $\mathrm{GrI}^{\text {hi }}$ monocytes in liver fibrosis. (a) Gating strategy of Gr1 ${ }^{\text {hi }}$ monocytes for flow cytometric analysis. (b) Proportions of $\mathrm{Gr}^{\mathrm{hi}}$ monocytes in the liver tissues. All data are expressed as the mean \pm SEM. ${ }^{*} P<0.05 ;{ }^{* *} P<0.01$. Original magnification: $\times 400$; bar $=200 \mu \mathrm{m}$.

suppressing the expression of matrix metalloproteinases [40]. TGF- $\beta 1$ mainly comes from monocyte-derived macrophages $[29,40]$. GRNs is a family of protein growth factors that are involved in cell proliferation. Macrophage-secreted granulin may activate resident HSCs into myofibroblasts resulting in a fibrotic microenvironment [7]. In our present study, we found that the hepatic expression of proinflammatory and profibrogenic cytokines IL-1 $\beta$, IL-6, TNF- $\alpha$, TGF- $\beta 1$, and GRN was significantly increased following $\mathrm{CCl}_{4}$ injection. However, emodin administration was able to decrease the hepatic expression of IL- $1 \beta$, TNF- $\alpha$, GRN, and TGF- $\beta 1$ in the liver fibrosis model, which also explains why emodin can alleviate liver inflammation and fibrosis.

CCL7 and MCP-1 are the major cytokines for the recruitment of $\mathrm{Gr}{ }^{\text {hi }}$ monocytes in the liver [41, 42]. MCP-1 has been well studied in liver fibrosis. In acute and chronic liver injury, MCP-1 can induce liver fibrosis through recruitment of $\mathrm{Gr}^{\mathrm{hi}}$ monocytes [28]. In the present study, we also found that the hepatic expression of MCP-1 was significantly higher in the $\mathrm{CCl}_{4}$ group as compared with the control group. In $\mathrm{Balb} / \mathrm{C}$ mice fed on the methionine/choline-deficient diet, a lack of MCP-1 was associated with lower ALT levels and 


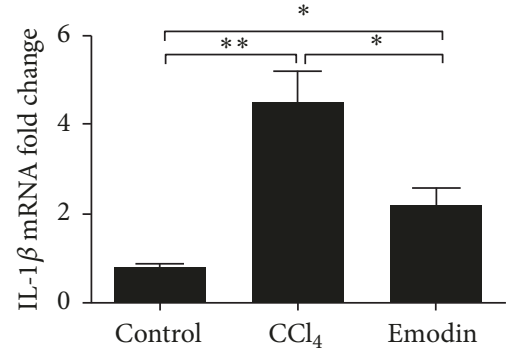

(a)

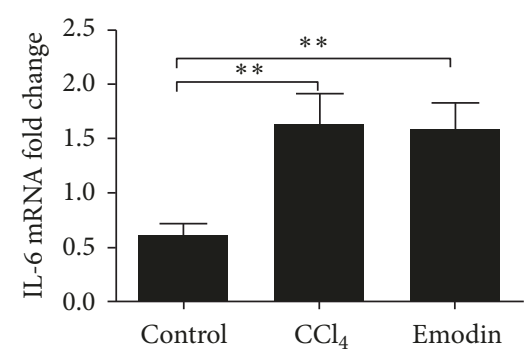

(b)

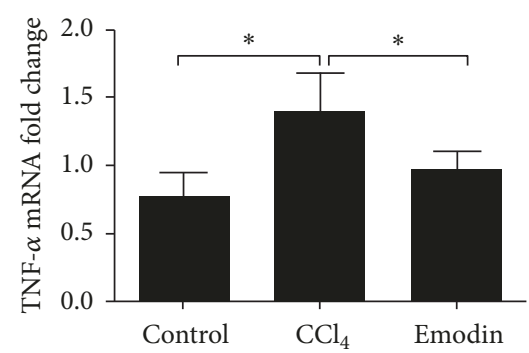

(c)

FIGURE 5: Emodin inhibited $G r 1^{\text {hi }}$ monocyte associated proinflammatory cytokines. All data are expressed as the mean \pm SEM. ${ }^{* *} P<0.01$; ${ }^{*} P<0.05$.
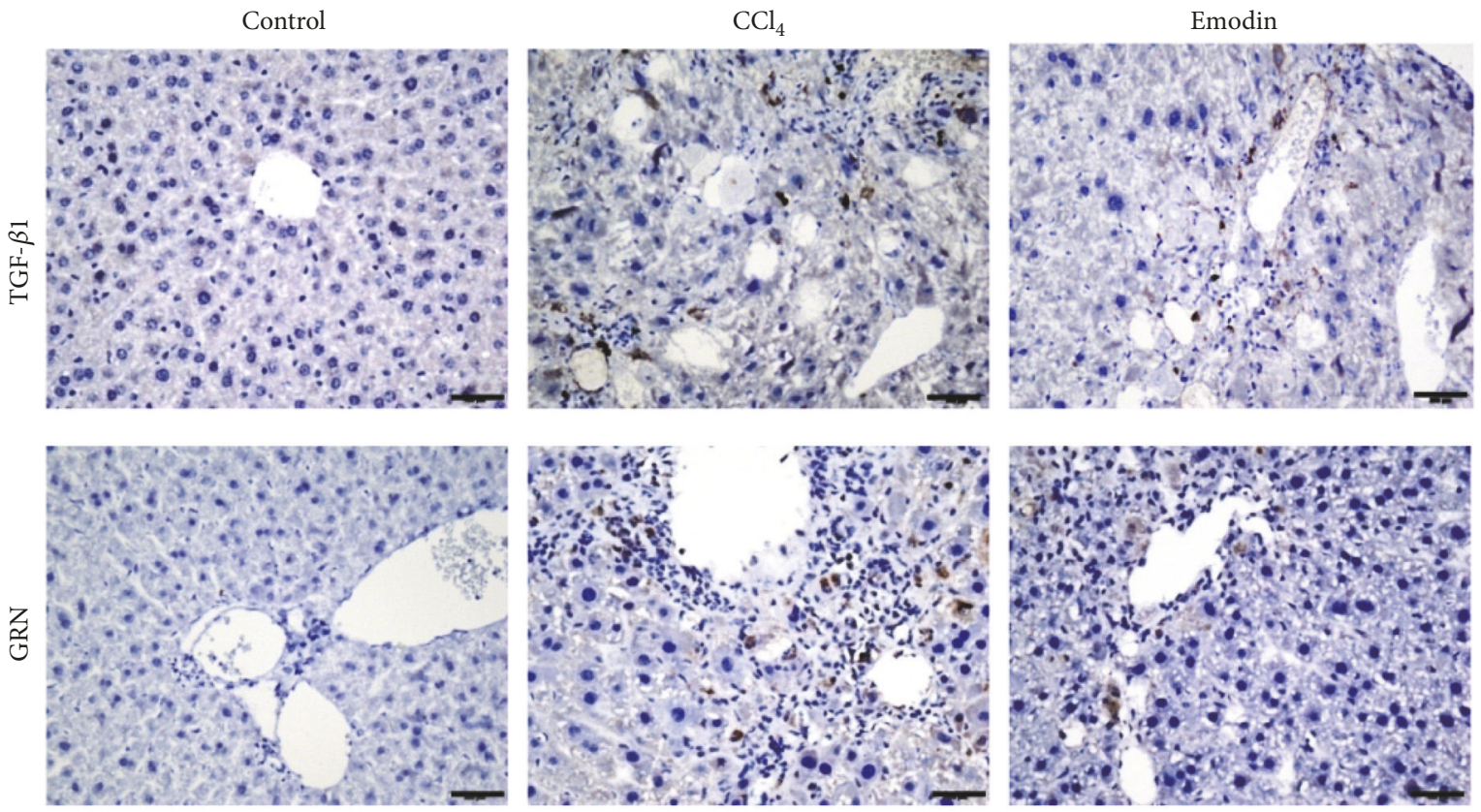

(a)
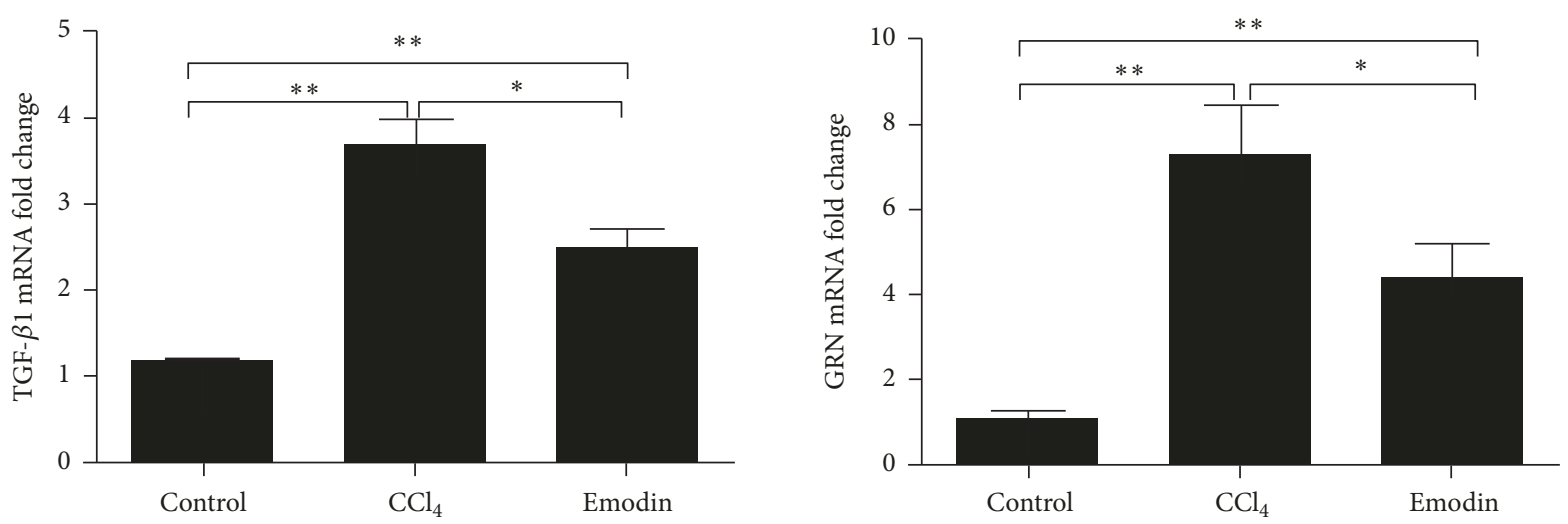

(b)

FIGURE 6: Emodin inhibited the Gr1 ${ }^{\text {hi }}$ monocyte associated profibrogenic cytokines. (a) Immunohistochemical staining of GRN and TGF- $\beta 1$ in the liver tissues. (b) Hepatic mRNA expression of GRN and TGF- $\beta 1$ in the liver tissues. All data are expressed as the mean \pm SEM. ${ }^{* *} P<0.01$; ${ }^{*} P<0.05$. Original magnification: $\times 400$; bar $=200 \mu \mathrm{m}$. 

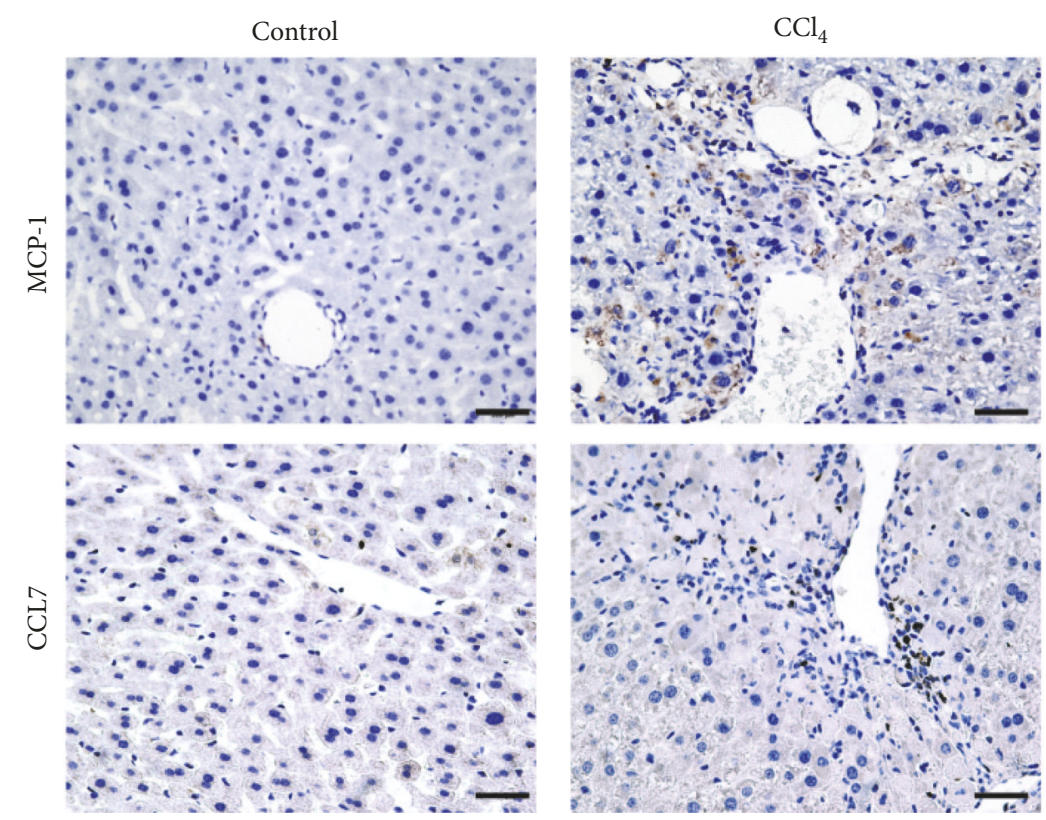

(a)
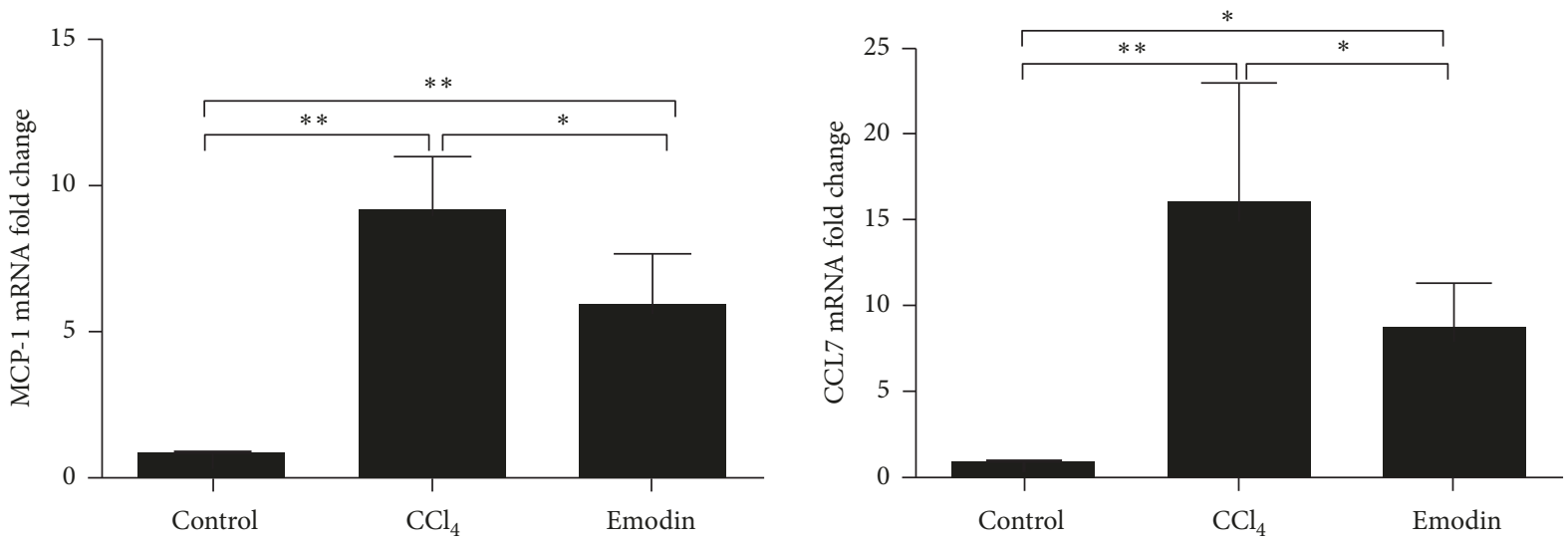

(b)

FIGURE 7: Emodin inhibited the expression of chemokines MCP-1 and CCL7. (a) Immunohistochemical staining of MCP-1 and CCL7 in the liver tissues. (b) Hepatic mRNA expression of MCP-1 and CCL7. All data are expressed as the mean \pm SEM. ${ }^{* *} P<0.01 ;{ }^{*} P<0.05$. Original magnification: $\times 400$; bar $=200 \mu \mathrm{m}$.

reduced infiltration of inflammatory cells, together with a lower degree of liver fibrosis [43]. Pharmacological inhibition of MCP-1 by mNOX-E36 can also significantly reduce the infiltration of $\mathrm{Gr}^{\text {hi }}$ monocytes in the process of acute and chronic liver injury and can reduce the degree of liver fibrosis in vivo [21, 30,44]. CCL7, previously known as monocyte chemotactic protein-3, belongs to the MCP subfamily of CCLs. CCL7 is expressed at multiple sites of inflammation and is produced by monocytes, fibroblasts, endothelial cells, and mast cells [45-48]. Compared to MCP1-deficient mice, CCL7-deficient mice showed less infiltration of monocytes in the encephalitis model caused by West Nile virus infection, which indicates that CCL7 has a stronger chemotactic activity than that of MCP-1 [49]. Inhibition of CCL7 could significantly reduce the infiltration of monocytes/macrophages to lung tissues of mice infected

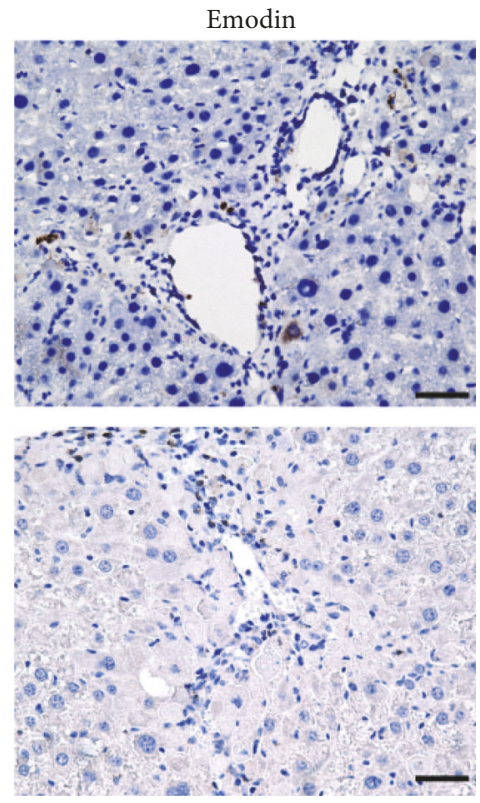




\section{Conflicts of Interest}

The authors have declared that no conflicts of interest exist regarding the publication of this paper.

\section{Authors' Contributions}

Xiang-An Zhao and Guangmei Chen contributed equally to this work.

\section{Acknowledgments}

The study was supported from the National Natural Science Foundation of China (81672025 and 81702011), Medical Science and Technology Development Foundation of Nanjing (ZDX16004 and YKK16118), Nanjing Science and Technology Development Plan (201503016), Jiangsu Provincial Medical Innovation Team (CXTDA2017005), Natural Science Foundation of Jiangsu Province for Young Scholar (BK20160121), and Nanjing Medical Science and Technique Development Foundation (QRX17121).

\section{References}

[1] R. Bataller and D. A. Brenner, "Liver fibrosis," The Journal of Clinical Investigation, vol. 115, no. 2, pp. 209-218, 2005.

[2] A. Altamirano-Barrera, B. Barranco-Fragoso, and N. MéndezSánchez, "Management strategies for liver fibrosis," Annals of Hepatology, vol. 16, no. 1, pp. 48-56, 2017.

[3] J. Guo and S. L. Friedman, "Hepatic fibrogenesis," Seminars in Liver Disease, vol. 27, no. 4, pp. 413-426, 2007.

[4] A. Adhyatmika, K. S. Putri, L. Beljaars, and B. N. Melgert, "The Elusive Antifibrotic Macrophage," Frontiers in Medicine, vol. 2, article 81, 2015.

[5] C. Ju and F. Tacke, "Hepatic macrophages in homeostasis and liver diseases: from pathogenesis to novel therapeutic strategies," Cellular \& Molecular Immunology, vol. 13, no. 3, pp. 316-327, 2016.

[6] M. W. Robinson, C. Harmon, and C. O'Farrelly, "Liver immunology and its role in inflammation and homeostasis," Cellular \& Molecular Immunology, vol. 13, no. 3, pp. 267-276, 2016.

[7] S. R. Nielsen, V. Quaranta, A. Linford et al., "Macrophagesecreted granulin supports pancreatic cancer metastasis by inducing liver fibrosis," Nature Cell Biology, vol. 18, no. 5, pp. 549-560, 2016.

[8] K. R. Karlmark, R. Weiskirchen, and H. W. Zimmermann, "Hepatic recruitment of the inflammatory $\mathrm{Grl}^{+}$monocyte subset upon liver injury promotes hepatic fibrosis," Hepatology, vol. 50, no. 1, pp. 261-274, 2009.

[9] J. Pradere, J. Kluwe, S. de Minicis et al., "Hepatic macrophages but not dendritic cells contribute to liver fibrosis by promoting the survival of activated hepatic stellate cells in mice," Hepatology, vol. 58, no. 4, pp. 1461-1473, 2013.

[10] E. Liaskou, H. W. Zimmermann, K.-K. Li et al., "Monocyte subsets in human liver disease show distinct phenotypic and functional characteristics," Hepatology, vol. 57, no. 1, pp. 385398, 2013.

[11] H. W. Zimmermann, S. Seidler, J. Nattermann et al., "Functional contribution of elevated circulating andhepatic non-classical
CD14+CD16+ monocytes to inflammation and human liver fibrosis," PLoS ONE, vol. 5, no. 6, Article ID el1049, 2010.

[12] R. Weiskirchen and F. Tacke, "Liver Fibrosis: From Pathogenesis to Novel Therapies," Digestive Diseases, vol. 34, no. 4, pp. 410422, 2016.

[13] T. A. Wynn and L. Barron, "Macrophages: master regulators of inflammation and fibrosis," Seminars in Liver Disease, vol. 30, no. 3, pp. 245-257, 2010.

[14] M. Melino, V. L. Gadd, K. A. Alexander et al., "Spatiotemporal characterization of the cellular and molecular contributors to liver fibrosis in a murine hepatotoxic-injury model," The American Journal of Pathology, vol. 186, no. 3, pp. 524-538, 2016.

[15] L. Beattie, A. Sawtell, J. Mann et al., "Bone marrow-derived and resident liver macrophages display unique transcriptomic signatures but similar biological functions," Journal of Hepatology, vol. 65, no. 4, pp. 758-768, 2016.

[16] C. L. Scott, F. Zheng, P. De Baetselier et al., "Bone marrowderived monocytes give rise to self-renewing and fully differentiated Kupffer cells," Nature Communications, vol. 7, Article ID 10321, 2016.

[17] K. J. Brempelis and I. N. Crispe, "Infiltrating monocytes in liver injury and repair," Clinical \& Translational Immunology, vol. 5, no. 11, article el13, 2016.

[18] E. Seki, S. de Minicis, and S. Inokuchi, "CCR2 promotes hepatic fibrosis in mice," Hepatology, vol. 50, no. 1, pp. 185-197, 2009.

[19] M. Imamura, T. Ogawa, Y. Sasaguri, K. Chayama, and H. Ueno, "Suppression of macrophage infiltration inhibits activation of hepatic stellate cells and liver fibrogenesis in rats," Gastroenterology, vol. 128, no. 1, pp. 138-146, 2005.

[20] C. Mitchell, D. Couton, J.-P. Couty et al., "Dual role of CCR2 in the constitution and the resolution of liver fibrosis in mice," The American Journal of Pathology, vol. 174, no. 5, pp. 1766-1775, 2009.

[21] J. Ehling, M. Bartneck, X. Wei et al., "CCL2-dependent infiltrating macrophages promote angiogenesis in progressive liver fibrosis," Gut, vol. 63, no. 12, pp. 1960-1971, 2014.

[22] F. Tacke and H. W. Zimmermann, "Macrophage heterogeneity in liver injury and fibrosis," Journal of Hepatology, vol. 60, no. 5, pp. 1090-1096, 2014.

[23] S. You, M. Zhou, B. Xue et al., "A clinical study on bing gan ling oral liquid for treatment of hepatitis C," J. Tradit. Chin. Med, vol. 18, no. 3, pp. 209-214, 1998.

[24] X. Dong, J. Fu, X. Yin et al., "Emodin: A Review of its Pharmacology, Toxicity and Pharmacokinetics," Phytotherapy Research, vol. 30, no. 8, pp. 1207-1218, 2016.

[25] W.-F. Lin, C. Wang, and C.-Q. Ling, "Research progress in antitumor effect of emodin," Zhongguo Zhongyao Zazhi, vol. 40, no. 20, pp. 3937-3940, 2015.

[26] R. Gao, R. Chen, Y. Cao et al., "Emodin suppresses TGF- $\beta 1$ induced epithelial-mesenchymal transition in alveolar epithelial cells through Notch signaling pathway," Toxicology and Applied Pharmacology, vol. 318, pp. 1-7, 2017.

[27] M.-X. Dong, Y. Jia, Y.-B. Zhang et al., "Emodin protects rat liver from CCl4-induced fibrogenesis via inhibition of hepatic stellate cells activation," World Journal of Gastroenterology, vol. 15, no. 38, pp. 4753-4762, 2009.

[28] K. Miura, L. Yang, N. van Rooijen, H. Ohnishi, and E. Seki, "Hepatic recruitment of macrophages promotes nonalcoholic steatohepatitis through CCR2," American Journal of PhysiologyGastrointestinal and Liver Physiology, vol. 302, no. 11, pp. G1310G1321, 2012. 
[29] A. Sica, P. Invernizzi, and A. Mantovani, "Macrophage plasticity and polarization in liver homeostasis and pathology," Hepatology, vol. 59, no. 5, pp. 2034-2042, 2014.

[30] C. Baeck, X. Wei, M. Bartneck et al., "Pharmacological inhibition of the chemokine $\mathrm{C}$-C motif chemokine ligand 2 (monocyte chemoattractant protein 1) accelerates liver fibrosis regression by suppressing Ly-6C+ macrophage infiltration in mice," Hepatology, vol. 59, no. 3, pp. 1060-1072, 2014.

[31] X. Li, Z. Xu, S. Wang et al., "Emodin ameliorates hepatic steatosis through endoplasmic reticulum-stress sterol regulatory element-binding protein 1c pathway in liquid fructosefeeding rats," Hepatology Research, vol. 46, no. 3, pp. E105-E117, 2016.

[32] Y. Liu, X. Chen, M. Qiu, W. Chen, Z. Zeng, and Y. Chen, "Emodin ameliorates ethanol-induced fatty liver injury in mice," Pharmacology, vol. 94, pp. 71-77, 2014.

[33] T.-L. Pan, P.-W. Wang, C.-H. Huang et al., "Herbal formula, Scutellariae radix and Rhei rhizoma attenuate dimethylnitrosamine-induced liver fibrosis in a rat model," Scientific Reports, vol. 5, Article ID 11734, 2015.

[34] S.-K. Heo, H.-J. Yun, E.-K. Noh, and S.-D. Park, "Emodin and rhein inhibit LIGHT-induced monocytes migration by blocking of ROS production," Vascular Pharmacology, vol. 53, no. 1-2, pp. 28-37, 2010.

[35] M. Engele, E. Stößel, K. Castiglione et al., "Induction of TNF in human alveolar macrophages as a potential evasion mechanism of virulent Mycobacterium tuberculosis," The Journal of Immunology, vol. 168, no. 3, pp. 1328-1337, 2002.

[36] H. Ito, K. Ando, T. Ishikawa et al., "Role of TNF- $\alpha$ produced by nonantigen-specific cells in a fulminant hepatitis mouse model," The Journal of Immunology, vol. 182, no. 1, pp. 391-397, 2009.

[37] H. W. Zimmermann, S. Seidler, N. Gassler et al., "Interleukin-8 is activated in patients with chronic liver diseases and associated with hepatic macrophage accumulation in human liver fibrosis," PLoS ONE, vol. 6, no. 6, Article ID e21381, 2011.

[38] V. Hernandez-Gea and S. L. Friedman, "Pathogenesis of liver fibrosis," Annual Review of Pathology: Mechanisms of Disease, vol. 6, pp. 425-456, 2011.

[39] Y. Inagaki, R. Higashiyama, and K. Higashi, "Novel anti-fibrotic modalities for liver fibrosis: molecular targeting and regenerative medicine in fibrosis therapy," Journal of Gastroenterology and Hepatology, vol. 27, supplement 2, pp. 85-88, 2012.

[40] F. Heymann, L. Hammerich, D. Storch et al., "Hepatic macrophage migration and differentiation critical for liver fibrosis is mediated by the chemokine receptor $\mathrm{C}-\mathrm{C}$ motif chemokine receptor 8 in mice," Hepatology, vol. 55, no. 3, pp. 898-909, 2012.

[41] W. Xuan, Q. Qu, B. Zheng, S. Xiong, and G.-H. Fan, "The chemotaxis of M1 and M2 macrophages is regulated by different chemokines," Journal of Leukocyte Biology, vol. 97, no. 1, pp. 6169, 2015.

[42] F. Marra and F. Tacke, "Roles for chemokines in liver disease," Gastroenterology, vol. 147, no. 3, pp. 577-594, 2014.

[43] S. Galastri, E. Zamara, S. Milani et al., "Lack of CC chemokine ligand 2 differentially affects inflammation and fibrosis according to the genetic background in a murine model of steatohepatitis," Clinical Science, vol. 123, no. 7, pp. 459-471, 2012.

[44] C. Baeck, A. Wehr, K. R. Karlmark et al., "Pharmacological inhibition of the chemokine CCL2 (MCP-1) diminishes liver macrophage infiltration and steatohepatitis in chronic hepatic injury," Gut, vol. 61, no. 3, pp. 416-426, 2012.
[45] P. Menten, A. Wuyts, and J. Van Damme, "Monocyte chemotactic protein-3," European Cytokine Network, vol. 12, no. 4, pp. 554-560, 2001.

[46] J. W. Cheng, Z. Sadeghi, A. D. Levine et al., "The role of CXCL12 and CCL7 chemokines in immune regulation, embryonic development, and tissue regeneration," Cytokine, vol. 69, no. 2, pp. 277-283, 2014.

[47] P. Proost, A. Wuyts, and J. Van Damme, "Human monocyte chemotactic proteins-2 and -3: Structural and functional comparison with MCP-1," Journal of Leukocyte Biology, vol. 59, no. 1, pp. 67-74, 1996.

[48] B. Jacobsson, R.-M. Holst, B. Andersson, and H. Hagberg, "Monocyte chemotactic protein-2 and -3 in amniotic fluid: Relationship to microbial invasion of the amniotic cavity, intraamniotic inflammation and preterm delivery," Acta Obstetricia et Gynecologica Scandinavica, vol. 84, no. 6, pp. 566-571, 2005.

[49] S. V. Bardina, D. Michlmayr, K. W. Hoffman et al., "Differential roles of chemokines CCL2 and CCL7 in monocytosis and leukocyte migration during West Nile virus infection," The Journal of Immunology, vol. 195, no. 9, pp. 4306-4318, 2015.

[50] J. Girkin, L. Hatchwell, P. Foster et al., "CCL7 and IRF-7 mediate hallmark inflammatory and IFN responses following rhinovirus 1B infection," The Journal of Immunology, vol. 194, no. 10, pp. 4924-4930, 2015. 


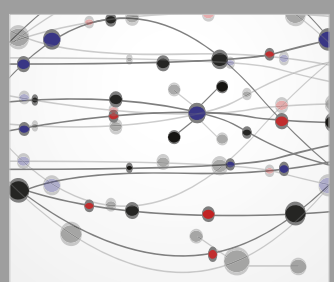

The Scientific World Journal
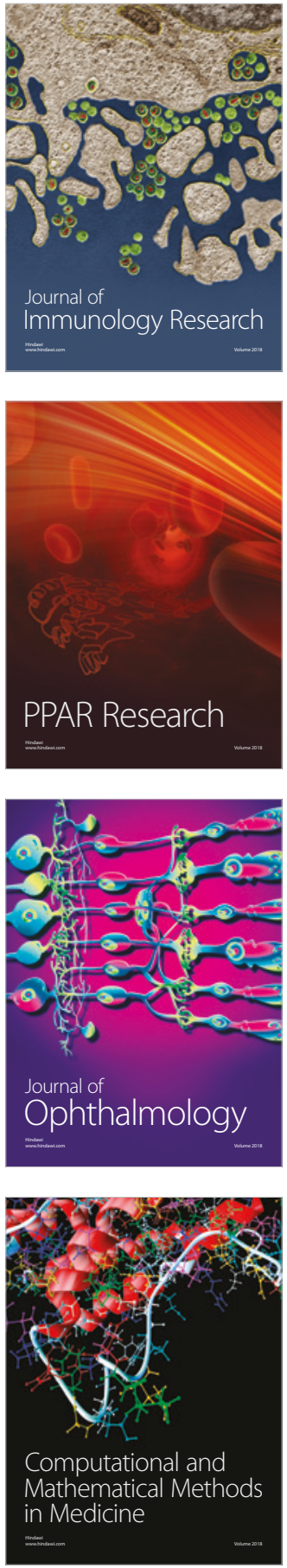

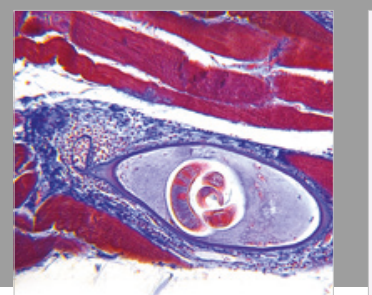

Gastroenterology Research and Practice

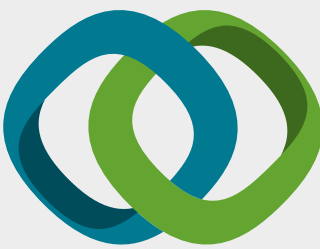

\section{Hindawi}

Submit your manuscripts at

www.hindawi.com
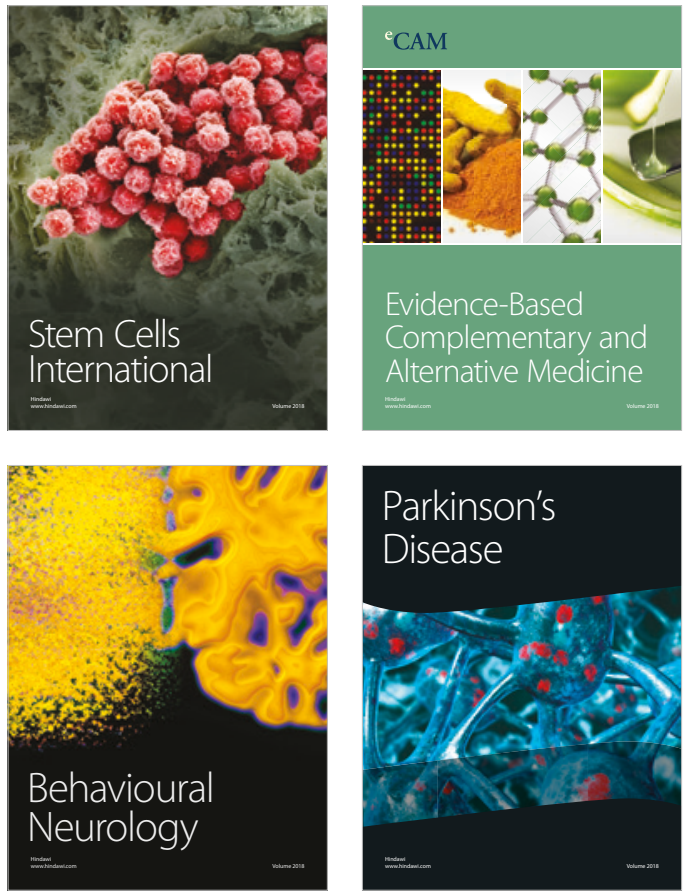

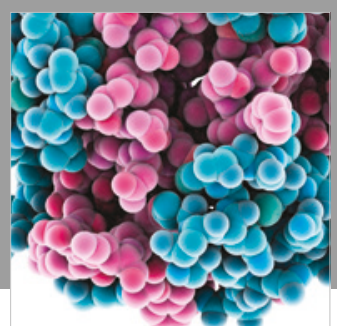

ournal of

Diabetes Research

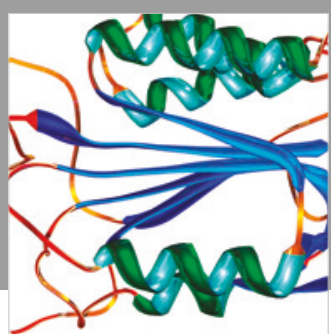

Disease Markers
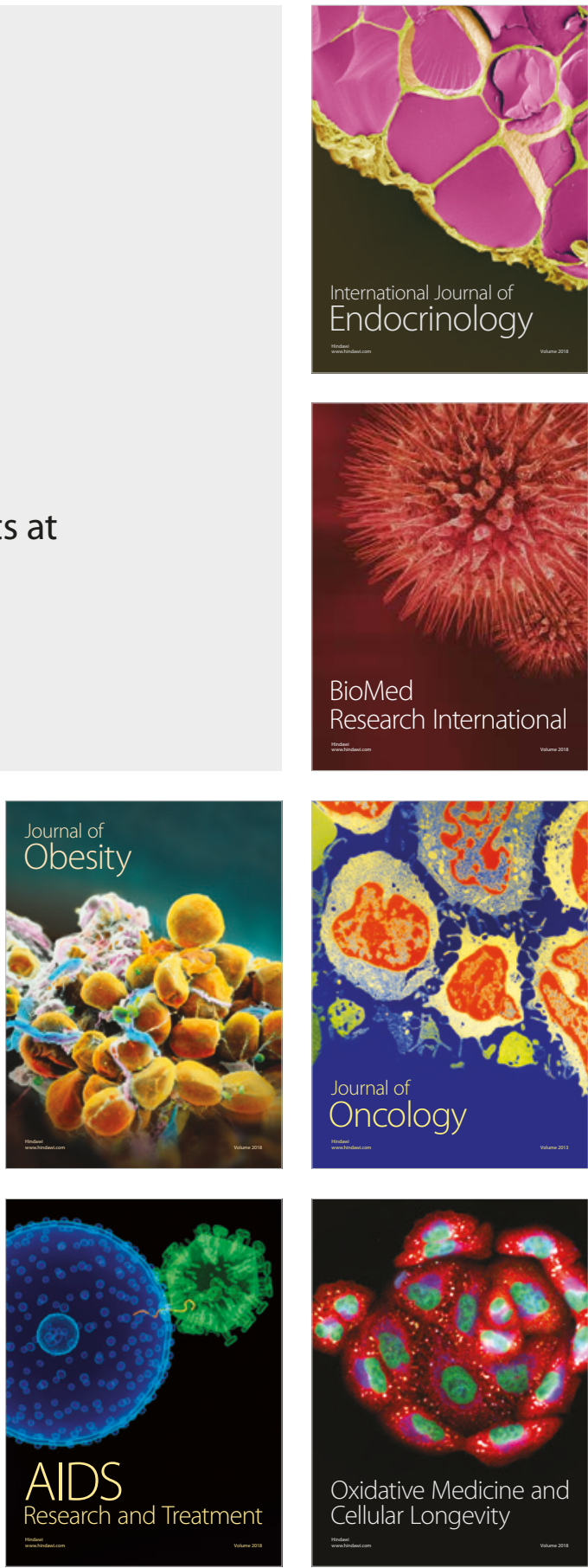